\title{
Recent Advances in the Detection of Neonatal Candidiasis
}

\author{
Laura Corbin Downey • P. Brian Smith • \\ Daniel K. Benjamin Jr. • Michael Cohen-Wolkowiez
}

Published online: 25 February 2010

(C) Springer Science+Business Media, LLC 2010

\begin{abstract}
Neonatal candidiasis is serious and often fatal. Blood culture, the standard for diagnosis, has a sensitivity of $50 \%$ or less, and isolate speciation and susceptibility takes several days. This review explores recent advances in Candida detection using various diagnostic strategies.
\end{abstract}

\section{Keywords Diagnosis $\cdot$ Neonate $\cdot$ Candida}

\section{Introduction}

Neonatal candidiasis is a common complication in extremely preterm infants surviving beyond the first 2 weeks of life [1]. Approximately $10 \%$ of extremely low birth weight $(<1,000 \mathrm{~g}$ birth weight) neonates develop candidiasis [2-4], and candidemia accounts for $12-15 \%$ of late-onset sepsis episodes in the neonatal intensive care unit (NICU) [1, 5]. Neonates diagnosed with candidiasis are at significant risk for mortality (20\%) [1, 2, 6, 7] and morbidity among survivors, including retinopathy of prematurity, chronic lung disease, periventricular leukomalacia, and poor longterm neurodevelopmental outcomes [1, 2, 7-11].

Risk factors for invasive candidiasis in neonates include prematurity, broad-spectrum antibiotics (e.g., third-generation

L. C. Downey • P. B. Smith • D. K. Benjamin Jr. •

M. Cohen-Wolkowiez

Department of Pediatrics and Duke Clinical Research Institute,

Duke University Medical Center,

Durham, NC, USA

D. K. Benjamin Jr.

e-mail: danny.benjamin@duke.edu

P. B. Smith $(\triangle)$

Duke Clinical Research Institute,

Box 17969, Durham, NC 27715, USA

e-mail: brian.smith@duke.edu cephalosporins) $[2,3]$, histamine-2 receptor blockers [4, 12], postnatal steroids [13-15], and presence of an indwelling catheter [4, 5]. In addition, the underdeveloped immune system of the premature neonate lacks basic immunologic functions such as chemotaxis, cytokine production, production of antibodies, and phagocytosis [16].

Although the devastating consequences of neonatal invasive candidiasis have been clearly identified, early detection of candidiasis remains a challenge. More rapid, accurate, and reliable methods are needed to diagnose neonatal candidiasis. This article reviews recent advances in various methods aimed at detecting invasive candidiasis, with a special focus on the application of these methods to the neonatal population.

\section{Body Fluid Cultures}

Blood culture is the standard test for diagnosis of candidemia; however, this test confirms $50 \%$ or fewer of autopsy-proven cases of invasive candidiasis in adults [17]. In addition, this low sensitivity was observed in adults from whom more than $10 \mathrm{~mL}$ of blood was routinely collected for culture. In neonates, only $0.5-1 \mathrm{~mL}$ of blood is routinely available for culture, likely decreasing the sensitivity of the test substantially. In addition, even when a pathogen is isolated from blood culture, speciation and susceptibility testing can take several days. In a retrospective study of hospitalized patients ( 1 month to 65 years of age), there were 115 episodes of Candida bloodstream infections [18]. The mean time to detection was $35 \mathrm{~h}$ and $80 \mathrm{~h}$ for C. albicans and C. glabrata, respectively $(P<0.0001)$, and the mean time to final speciation was $86 \mathrm{~h}$ and $154 \mathrm{~h}$, respectively $(P<0.0001)$.

While the diagnosis of candidemia via blood culture is lengthy, techniques such as peptide nucleic acid fluorescence in situ hybridization used in conjunction with blood 
cultures may shorten the time to preliminary species identification [19]. Once a blood culture is positive for Candida, this test can rapidly (within $2 \mathrm{~h}$ ) identify up to five different Candida species with the use of fluorescentlabeled peptide nucleic acid probe. Although this test is potentially useful clinically, it requires a positive blood culture, lacks specificity, and is not able to fully distinguish between all clinically relevant Candida species.

\section{Hematologic Markers}

Hematologic parameters are often used to predict the presence of infection in neonates. However, the white blood cell count is not a useful aid in the diagnosis of Candida infections; as many as $40 \%$ of neonates with candidiasis have normal white blood cell counts [20].

Thrombocytopenia is another commonly used hematologic marker for candidiasis, but it lacks sensitivity and specificity. A single-center study found that thrombocytopenia was present in $84 \%$ of very-low-birth-weight (VLBW) neonates $(<1,500 \mathrm{~g}$ birth weight) with candidemia [21]. However, in a second study of VLBW neonates, thrombocytopenia was only $20 \%$ sensitive for the presence of candidemia [22] and $16 \%$ sensitive for the presence of bacteremia. The mean platelet nadir was $66,500 / \mathrm{mm}^{3}$ for Gram-positive sepsis, $46,000 / \mathrm{mm}^{3}$ for Gram-negative, and $38,000 / \mathrm{mm}^{3}$ for fungal sepsis.

\section{Fungal Antigens}

The two fungal antigens used most frequently for diagnostic purposes are $1->3 \beta$-D glucan (BG) and mannan (Table 1). Mannan is a high-molecular-weight polysaccharide present in the cell wall of Candida, and BG is an important structural component of the fungal cell wall. Two commonly used assays for mannan antigen detection include the Platelia Candida Ag (BioRad, Manes, La Coquette, France) and the Serion ELISA antigen Candida assay (Institute Virion/Serion, Wurzburg, Germany), which rely on monoclonal anti-mannan antibodies for detection [23]. Normal result values for these are $<0.25 \mathrm{ng} / \mathrm{mL}$ and $>1.15 \mathrm{U} / \mathrm{mL}$, respectively. The Fungitell test kit (Associates of Cape Cod, East Falmouth, MA) is an antigen assay used to detect BG concentrations in an unknown sample based on a known calibration curve. A negative result has a value $<60 \mathrm{pg} / \mathrm{mL}$ [23].

A study of 70 neonates admitted to the NICU with at least three mannan samples collected showed that the Platelia Candida mannan assay had an overall sensitivity and specificity of $94 \%$, and a culture-proven sensitivity and specificity of $92 \%$ and $84 \%$, respectively [24•]. These neonates were divided into two groups: those with proven $(n=12)$ or probable $(n=6)$ candidiasis (mean birth weight $=$ $1,622 \mathrm{~g})$, and those without candidiasis $(n=52$; mean birth weight $=2,262 \mathrm{~g}$ ). The antigen test was considered positive if there were two or more samples $\geq 0.5 \mathrm{ng} / \mathrm{mL}$. Twelve neonates had proven candidiasis by positive blood culture and clinical sepsis; $11(92 \%)$ of these had positive mannan assays. Of the remaining 58 neonates with negative blood cultures, nine (16\%) had a positive antigen assay; six $(67 \%)$ were considered to have invasive candidiasis based on a clinical diagnosis and lack of response to antibacterial treatment, while the other three were considered false positives. The mannan test was positive at a median of 8 days (range, 4-18 days) before blood culture.

A study of 51 patients ( $42 \%$ with invasive candidiasis) aged 3-65 years admitted to a tertiary care hospital for chemotherapy treatment evaluated Platelia Candida antigen assay (mannan) and Platelia Candida Ab/Ac/Ak assay (anti-mannan) levels before clinical evidence of disease [25]. Both mannan antigen and anti-mannan antibodies were detected before clinical evidence of candidiasis was apparent. Anti-mannan antibodies were detected significantly more frequently in patients who had experienced more than 15 days of neutropenia, compared with those with less than 15 days $(P<0.05)$. Compared with controls (patients receiving chemotherapy without candidiasis), mannan antigen was detected more frequently in patients with candidiasis in the first 15 neutropenic days (odds ratio $=3.7$; 95\% CI=1.4-9.7, $P<0.05$ ).

A follow-up study compared the use of several assays to detect mannan, anti-mannan antibodies, or BG in 21 neutropenic patients receiving chemotherapy with cultureproven invasive candidiasis and 30 controls without candidiasis undergoing myeloablative chemotherapy [23]. When using the Serion assay, the Candida mannan antigen and Candida anti-mannan antibodies were detected in 15/21 (71\%) and 9/21 (43\%) patients with candidiasis, compared with $7 / 30(23 \%)$ and $3 / 30(10 \%)$ controls, respectively. BG was detected in 16/21 (76\%) patients with candidiasis, compared with 12/30 (40\%). Overall, these tests produced a high number of false-positive results, leading to specificities ranging from $60 \%$ to $90 \%$. The sensitivities were slightly better (ranging from 70\%-79\%), with the exception of the anti-mannan antibody test (sensitivity of $43 \%$ ). Candida antigens were detected at a median of 11 days prior to culture confirmation of infection, with a range of 176 days pre- to 195 days post-culture confirmation, and antibodies were detected at a median of 20 days prior to culture confirmation (range of 197 days pre- to 8 days postculture confirmation). These studies demonstrate that, at least in neutropenic patients, the timing of an infection may determine the most reliable diagnostic test.

A study of four antigen tests (mannan [Platelia Candida antigen], anti-mannan antibodies [Platelia Candida anti- 
Table 1 Overview of diagnostic tools available for the diagnosis of candidemia

\begin{tabular}{|c|c|c|c|c|c|}
\hline & Study population & Patients, $n$ & $\begin{array}{l}\text { Sample } \\
\text { volume }\end{array}$ & Sensitivity & Specificity \\
\hline Blood culture [17] & Adults & 37 & & $50 \%^{\mathrm{a}}$ & $100 \%$ \\
\hline \multicolumn{6}{|l|}{ Fungal antigens } \\
\hline Mannan [24•] & Neonates & 70 & $300 \mu \mathrm{L}$ & $92 \%$ & $84 \%$ \\
\hline Mannan [23] & Chemotherapy & 51 & & $71 \%$ & $77 \%$ \\
\hline$[26]$ & Children and adults & 92 & $300 \mu \mathrm{L}$ & $41 \%$ & $100 \%$ \\
\hline $1->3 \beta$-D glucan [23] & Chemotherapy & 51 & & $76 \%$ & $60 \%$ \\
\hline $1->3 \beta$-D glucan $[26]$ & Children and adults & 92 & $500 \mu \mathrm{L}$ & $47 \%$ & $100 \%$ \\
\hline \multicolumn{6}{|l|}{ Fungal antibodies } \\
\hline Anti-mannan [23] & Chemotherapy & 51 & & $43 \%$ & $90 \%$ \\
\hline Anti-mannan [26] & Children and adults & 92 & & $47 \%$ & $100 \%$ \\
\hline \multicolumn{6}{|l|}{ PCR } \\
\hline Semi-nested [26] & Children and adults & 92 & & $88 \%$ & $100 \%$ \\
\hline Nested [28] & Hospitalized patients & 110 (24 neonates) & $200 \mu \mathrm{L}$ & $86 \%$ & $54 \%$ \\
\hline Real-time [28] & Hospitalized patients & 110 (24 neonates) & $200 \mu \mathrm{L}$ & $81 \%$ & $96 \%$ \\
\hline Real-time [31] & Inpatients & 23 & & $93 \%$ & $66 \%$ \\
\hline Real-time [31] & Inpatients & 23 & & $77 \%$ & $100 \%$ \\
\hline Real-time [32] & Immunocompromised & 384 & $2,500 \mu \mathrm{L}$ & $88 \%$ & $94 \%$ \\
\hline NASBA [37•] & Samples from positive blood cultures & 10 & & $100 \%$ & $100 \%$ \\
\hline
\end{tabular}

NASBA nucleic acid sequence-based amplification; $P C R$ polymerase chain reaction

${ }^{a}<50 \%$ sensitivity and $100 \%$ specificity in the clinical setting

body], BG [Fungitell], and semi-nested polymerase chain reaction [snPCR]) examined 109 blood samples from 92 children and adults: 27 hospitalized patients (aged 6 months to 88 years) with culture-proven candidemia ( 32 samples); 39 hospitalized patients with suspected candidemia (51 samples); 10 samples from outpatient women with $C$. albicans vaginitis; and 16 samples from healthy controls [26]. The sensitivity and specificity for each test were as follows: mannan assay $41 \%$ and $100 \%$, anti-mannan and BG $47 \%$ and $100 \%$, and snPCR $88 \%$ and $100 \%$, respectively. Testing for both mannan and anti-mannan antibodies increased the sensitivity to $75 \%$. The snPCR test, which allows for greater specificity compared with standard PCR by introducing a second primer within the amplified region from the first PCR reactions, identified five patients whose infection was due to more than one Candida species. Although fungal antigen tests are encouraging, they are expensive and not yet ready for use in standard practice (particularly in neonates, for whom there is little information on their performance).

\section{DNA PCR}

PCR is a process capable of identifying a specific target DNA sequence (primers) for a given pathogen using sequence amplification techniques. Nested PCR uses two sets of primers in order to minimize sample contamination. Real-time PCR uses fluorescent dyes to detect and quantify amplified DNA sequences as they are made during the PCR process, rather than afterward, which allows earlier DNA identification and target quantification.

Over the past few decades, PCR has been extensively used as a viral diagnostic test and is becoming a focus to discover faster and more accurate diagnostic methods for candidemia (Table 1). In addition to providing a more accurate diagnosis, PCR has the potential to identify the specific Candida species in a shorter amount of time and the ability to decrease the lower level of detection to less than ten colony-forming units per $\mathrm{mL}$ [27]. This level of detection is critical in the setting of low organism burden in the blood that is commonly seen with invasive candidiasis.

Two different PCR techniques, nested and real-time PCR, were compared in a study of 200 blood samples from 110 hospitalized patients (24 neonates) [28]. All samples were tested by blood culture, nested, and real-time PCR [28]. There were 36 positive blood cultures for yeast: 30 single Candida species, three mixed Candida, and three non-speciated yeasts. The sensitivity and specificity were $86 \%$ and $54 \%$ for nested PCR, and $81 \%$ and $96 \%$ for realtime PCR, respectively. 
Real-time PCR was used to compare the LightCycler SeptiFast PCR test (Roche Diagnostics, Penzberg, Germany) with the Bactec 9,240 blood culture system (Becton Dickinson, Heidelberg, Germany) [29]. The SeptiFast PCR test has the ability to identify 25 different bacterial or fungal pathogens (C. albicans, C. tropicalis, C. parapsilosis, C. krusei, C. glabrata, and Aspergillus fumigatus) [30]. The study examined 101 samples undergoing both diagnostic tests from 77 hospitalized adult patients (median age $=59$ years for those with positive PCR, 52 years for negative PCR) [29]. Of the eight patients with invasive fungal disease, two were both blood culture and PCR positive, two were blood culture positive and PCR negative, and four were blood culture negative and PCR positive. The sensitivity of the blood culture was only $50 \%$, compared with $75 \%$ for PCR.

A study of 58 serum samples from 23 patients used the LightCycler PCR system and two different fungal-specific primers, L18 and internal transcribed spacer (ITS) [31]. The control group included ten healthy volunteers. Thirteen of the $23(56.5 \%)$ patients had culture-proven candidemia. The L18 primer detected the presence of Candida DNA in $92 \%$ of positive cultures (12/13) but was not able to identify individual species. Use of the ITS primers allowed for the detection of Candida DNA in $77 \%$ of positive cultures (10/13), and the ITS primers allowed for rapid species differentiation between $C$. albicans, $C$. tropicalis, C. krusei, C. glabrata, and C. parapsilosis. The sensitivity and specificity of these two primers when used separately were $93 \%$ and $66 \%$ for L18 and $77 \%$ and $100 \%$ for the ITS primers, respectively. Although this assay may hold promise, it is not yet commercially available.

A three-center study compared blood cultures with realtime PCR using the MolYsis Complete5 kit (Molzym, Bremen, Germany) in 384 immunocompromised patients (including 55 children) with 468 infectious episodes [32]. The MolYsis Complete5 kit is used prior to PCR sample analysis to lyse white blood cells prior to bacterial or fungal cell wall destruction to maximize recovery of pathogen DNA. Seven of eight (88\%) episodes of culture-proven candidemia were PCR positive, and 28/460 (6\%) negative blood cultures had a positive PCR. The sensitivity of the PCR test combined with the use of the MolYsis kit was $88 \%$, with a specificity of $94 \%$. Positive PCR samples allowed for detection of fungal disease 3 days earlier than blood culture results.

PCR testing shows promise as a tool for the diagnosis of invasive candidiasis. The current limitations of this test include lack of probes that can detect multiple Candida species simultaneously, lack of specificity, potential for sample contamination leading to false-positive results, and difficult sample preparation. Additionally, these tests are understudied in the neonatal population.

\section{Nucleic Acid Sequence-Based Amplification}

Real-time nucleic acid sequence-based amplification (NASBA) is a technology used to amplify RNA from either RNA or DNA, as opposed to PCR, which amplifies only DNA. These tests are able to detect a target RNA without any background DNA contamination, decreasing the number of false-positive results. NASBA is a very sensitive diagnostic test with a lower level of detection of one colony-forming unit per $\mathrm{mL}$ [27], less than that required for PCR. NASBA has often been used to diagnose RNA viruses such as enterovirus [33, 34]. There are a few reported cases of it being used to diagnose fungal infections $[35,36]$, and no reports of its use in the diagnosis of bloodstream infections (Table 1).

Molecular beacons are hairpin probes used in conjunction with NASBA composed of nucleic acids that fluoresce once they are bound to their target [37•]. Investigators developed five molecular beacons and the corresponding primers to detect Gram-negative, Gram-positive, Candida, and Aspergillus organisms. The Candida primer was able to detect eight different species and the Aspergillus primer four species. Although there were few fungal blood samples $(n=10)$, the probes were found to be reliable with a sensitivity, specificity, positive predictive value, and negative predictive value of $100 \%$. However, due to the small number of samples, difficulty of use, and sparse amount of data, this test is not currently standard practice.

\section{Conclusions}

The accurate and timely diagnosis of invasive Candida disease in the neonatal population is challenging. However, recent focus has been aimed at investigating and improving alternative methods of diagnosis. Identification of a single colony of Candida in the blood is clinically significant. In spite of its poor sensitivity, blood culture continues to be the standard diagnostic test. While blood culture remains the current gold standard, other tests, such as fungal antigen tests, may currently be realistic adjunctive tests in specific populations. Real-time PCR and NASBA technologies show promise as diagnostic tools for patients in the NICU; however, they are currently understudied in this population. Accurate, rapid, and sensitive diagnosis of invasive Candida disease is needed for the neonatal population, in whom the burden of disease is high and outcomes are poor.

Disclosure Dr. Benjamin receives support from the United States Government for his work in pediatric and neonatal clinical pharmacology (1R01HD057956-02, 1R01FD003519-01, 1U10-HD45962-06, 1K24HD058735-01, and Government Contract HHSN267200700051C), 
the nonprofit organization Thrasher Research Foundation for his work in neonatal candidiasis (www.thrasherresearch.org), and from industry for neonatal and pediatric drug development (www.dcri.duke.edu/research/ coi.jsp). Dr. Smith received support from NICHD 1K23HD060040-01.

\section{References}

Papers of particular interest, published recently, have been highlighted as:

- Of importance

1. Stoll BJ, Hansen N, Fanaroff AA, et al.: Late-onset sepsis in very low birth weight neonates: the experience of the NICHD Neonatal Research Network. Pediatrics 2002, 110:285-291.

2. Benjamin DK Jr, Stoll BJ, Fanaroff AA, et al.: Neonatal candidiasis among extremely low birth weight infants: risk factors, mortality rates, and neurodevelopmental outcomes at 18 to 22 months. Pediatrics 2006, 117:84-92.

3. Cotten CM, McDonald S, Stoll B, et al.: The association of thirdgeneration cephalosporin use and invasive candidiasis in extremely low birth-weight infants. Pediatrics 2006, 118:717-722.

4. Saiman L, Ludington E, Pfaller M, et al.: Risk factors for candidemia in neonatal intensive care unit patients. The National Epidemiology of Mycosis Survey study group. Pediatr Infect Dis J 2000, 19:319-324.

5. Feja KN, Wu F, Roberts K, et al.: Risk factors for candidemia in critically ill infants: a matched case-control study. J Pediatr 2005, 147:156-161.

6. Stoll BJ, Gordon T, Korones SB, et al.: Late-onset sepsis in very low birth weight neonates: a report from the National Institute of Child Health and Human Development Neonatal Research Network. J Pediatr 1996, 129:63-71.

7. Benjamin DK, DeLong E, Cotten CM, et al.: Mortality following blood culture in premature infants: increased with Gram-negative bacteremia and candidemia, but not Gram-positive bacteremia. J Perinatol 2004, 24:175-180.

8. Stoll BJ, Hansen NI, Adams-Chapman I, et al.: Neurodevelopmental and growth impairment among extremely low-birth-weight infants with neonatal infection. JAMA 2004, 292:2357-2365.

9. Friedman S, Richardson SE, Jacobs SE, O'Brien K: Systemic Candida infection in extremely low birth weight infants: short term morbidity and long term neurodevelopmental outcome. Pediatr Infect Dis J 2000, 19:499-504.

10. Mittal M, Dhanireddy R, Higgins RD: Candida sepsis and association with retinopathy of prematurity. Pediatrics 1998, 101:654-657.

11. Kremer I, Naor N, Davidson S, et al.: Systemic candidiasis in babies with retinopathy of prematurity. Graefes Arch Clin Exp Ophthalmol 1992, 230:592-594.

12. Kaufman D: Strategies for prevention of neonatal invasive candidiasis. Semin Perinatol 2003, 27:414-424.

13. Malloy PJ, Zhao X, Madani ND, Feldman D: Cloning and expression of the gene from Candida albicans that encodes a highaffinity corticosteroid-binding protein. Proc Natl Acad Sci U S A 1993, 90:1902-1906.

14. Botas CM, Kurlat I, Young SM, Sola A: Disseminated candidal infections and intravenous hydrocortisone in preterm infants. Pediatrics 1995, 95:883-887.

15. Pera A, Byun A, Gribar S, et al.: Dexamethasone therapy and Candida sepsis in neonates less than 1250 grams. J Perinatol 2002, 22:204-208.

16. Chapman RL, Faix RG: Persistently positive cultures and outcome in invasive neonatal candidiasis. Pediatr Infect Dis J 2000, 19:822-827.
17. Berenguer J, Buck M, Witebsky F, et al.: Lysis-centrifugation blood cultures in the detection of tissue-proven invasive candidiasis. Disseminated versus single-organ infection. Diagn Microbiol Infect Dis 1993, 17:103-109.

18. Fernandez J, Erstad BL, Petty W, Nix DE: Time to positive culture and identification for Candida blood stream infections. Diagn Microbiol Infect Dis 2009, 64:402-407.

19. Shepard JR, Merz WG, Gherna M, et al.: Evaluation of yeast traffic light PNA FISH ${ }^{\mathrm{TM}}$ for detection of high prevalence Candida species. Poster presented at the 107th American Society for Microbiology General Meeting. Toronto, Canada; May 21-25, 2007.

20. Makhoul IR, Kassis I, Smolkin T, et al.: Review of 49 neonates with acquired fungal sepsis: further characterization. Pediatrics 2001, 107:61-66.

21. Guida JD, Kunig AM, Leef KH, et al.: Platelet count and sepsis in very low birth weight neonates: is there an organism-specific response? Pediatrics 2003, 111:1411-1415.

22. Manzoni P, Mostert M, Galletto P, et al.: Is thrombocytopenia suggestive of organism- specific response in neonatal sepsis? Pediatr Int 2009, 51:206-210.

23. Lunel FM, Mennink-Kersten MA, Ruegebrink D, et al.: Value of Candida serum markers in patients with invasive candidiasis after myeloablative chemotherapy. Diagn Microbiol Infect Dis 2009, 64:408-415.

24. - Oliveri S, Trovato L, Betta P, et al.: Experience with the Platelia Candida ELISA for the diagnosis of invasive candidosis in neonatal patients. Clin Microbiol Infect 2008, 14:391-393. This is one of the few recent studies on detection of neonatal candidiasis. While just a preliminary study, it suggests the possibility of mannan detection as a helpful tool in the diagnosis of candidemia in select neonates.

25. Verduyn Lunel FM, Donnelly JP, van der Lee HA, et al.: Circulating Candida-specific anti-mannan antibodies precede invasive candidiasis in patients undergoing myelo- ablative chemotherapy. Clin Microbiol Infect 2009, 15:380-386.

26. Alam FF, Mustafa AS, Khan ZU: Comparative evaluation of (1, 3)-beta-D-glucan, mannan and anti-mannan antibodies, and Candida species-specific snPCR in patients with candidemia. BMC Infect Dis 2007, 7:103.

27. Loeffler J, Hebart H, Cox P, et al.: Nucleic acid sequence-based amplification of Aspergillus RNA in blood samples. J Clin Microbiol 2001, 39:1626-1629.

28. Khlif M, Mary C, Sellami H, et al.: Evaluation of nested and realtime PCR assays in the diagnosis of candidaemia. Clin Microbiol Infect 2009, 15:656-661.

29. Dierkes C, Ehrenstein B, Siebig S, et al.: Clinical impact of a commercially available multiplex PCR system for rapid detection of pathogens in patients with presumed sepsis. BMC Infect Dis 2009, 9:126.

30. Lehmann LE, Hunfeld KP, Emrich T, et al.: A multiplex real-time PCR assay for rapid detection and differentiation of 25 bacterial and fungal pathogens from whole blood samples. Med Microbiol Immunol 2008, 197:313-324.

31. Dunyach C, Bertout S, Phelipeau C, et al.: Detection and identification of Candida spp. in human serum by LightCycler real-time polymerase chain reaction. Diagn Microbiol Infect Dis 2008, 60:263-271.

32. Wellinghausen N, Siegel D, Winter J, Gebert S: Rapid diagnosis of candidaemia by real- time PCR detection of Candida DNA in blood samples. J Med Microbiol 2009, 58:1106-1111.

33. Landry ML, Garner R, Ferguson D: Real-time nucleic acid sequence-based amplification using molecular beacons for detection of enterovirus RNA in clinical specimens. J Clin Microbiol 2005, 43:3136-3139.

34. Rutjes SA, Italiaander $\mathrm{R}$, van den Berg $\mathrm{HH}$, et al.: Isolation and detection of enterovirus RNA from large-volume water samples by using the NucliSens miniMAG system and real-time nucleic 
acid sequence-based amplification. Appl Environ Microbiol 2005, 71:3734-3740.

35. Borst A, Leverstein-Van Hall MA, Verhoef J, Fluit AC: Detection of Candida spp. in blood cultures using nucleic acid sequencebased amplification (NASBA). Diagn Microbiol Infect Dis 2001, 39:155-160.

36. Widjojoatmodjo MN, Borst A, Schukkink RA, et al.: Nucleic acid sequence-based amplification (NASBA) detection of med- ically important Candida species. J Microbiol Methods 1999, 38:81-90.

37. - Zhao Y, Park S, Kreiswirth BN, et al.: Rapid real-time nucleic acid sequence-based amplification-molecular beacon platform to detect fungal and bacterial bloodstream infections. J Clin Microbiol 2009, 47:2067-2078. This article uses a known technology in a new way; it holds some promise in becoming a useful tool in the diagnosis of candidemia. 